\title{
SURFACE EFFECTS IN SPIN-WAVE SPECTRUM OF THE SEMI-INFINITE $\mathrm{FeBr}_{2}$ FIELD-INDUCED METAMAGNET*
}

\author{
W. RUdZińSKI AND W. MACIEJEWSKI
}

Institute of Physics, A. Mickiewicz University, Matejki 48/49, 60-769 Poznań, Poland

Surface spin waves in the layered structure of the $\mathrm{FeBr}_{2}$ field-induced metamagnet are studied theoretically. Surface spin-wave modes are calculated for the (001) surface and for the paramagnetic phase at low temperatures. Allowing the surface exchange parameters to deviate from the bulk values, full richness of the surface spin-wave spectra is displayed. The problem of the stability of the ground state of the system is also discussed.

PACS numbers: 75.30.Ds, 75.30.Kz

\section{Introduction}

Recently, the semi-infinite layered structures of field-induced metamagnets (FIM) have been the subject of theoretical studies of their surface properties, and it has been shown that in addition to the bulk spin-wave excitations in low temperatures, $T \ll T_{\mathrm{N}}$, also surface spin waves (SSW) localized near the surface may occur in both antiferromagnetic (A) and paramagnetic (P) phases. The numerical results obtained in Ref. [1] for the (001) surface of the $\mathrm{FeBr}_{2}$ and $\mathrm{FeCl}_{2}$ FIM compounds revealed a strong dependence of SSW properties on the crystal structure of these materials. In Refs. [2] and [3] the effect of anisotropy on SSW has been investigated and consequently a modification of the frequency separation between the surface and bulk dispersion curves was obtained. This modification is more important in phase $\mathrm{P}$, as from the result of Ref. [1] it follows that in phase A the SSW in both $\mathrm{FeBr}_{2}$ and $\mathrm{FeCl}_{2}$ crystals are separated in frequency to a larger extent and thus they could be more readily resolved experimentally.

Moreover, by allowing the surface anisotropy parameter to deviate from the bulk value it has been found that in addition to the optical SSW predicted to exist in phase $\mathrm{P}$, also acoustic surface spin-wave branches may lie below the bulk spectrum.

In this paper we investigate the low temperature properties of SSW in the $\mathrm{FeBr}_{2}$ field-induced metamagnet for different values of surface exchange parameters. We shall limit our considerations to phase $\mathrm{P}$ and it will be shown that when

*This work was supported by the project 223519102 of the Committee for Scientific Research. 
the surface exchange parameters describing the coupling of spins parallel and normal to the crystal (001) surface are allowed to differ from their bulk value, a full richness of SSW features, including new physical solutions is displayed. Moreover, as a consequence of a competition between the surface intralayer and interlayer exchange interactions, the problem of the stability of the ground state of the system is under discussion.

\section{Model and method}

Let us consider a semi-infinite field-induced metamagnet as a system of ferromagnetically ordered layers parallel to the (001) surface. In phase A each layer is oriented in a direction opposite to that of the neighbouring layers. If the magnetic field $H$ is applied perpendicularly to the layers, at a threshold field $H_{\mathrm{AP}}$, a transition occurs to phase $\mathrm{P}$ of high magnetic moment. The system will be represented by the following Hamiltonian, known from Ref. [4] as the three-ion model of FIM:

$$
\begin{aligned}
\mathcal{H}= & -\frac{1}{2} \sum_{r, r^{\prime}, n} I_{r, r^{\prime}, n}\left(S_{r, n} \cdot S_{r^{\prime}, n}+\sigma^{\prime} S_{r, n}^{z} S_{r^{\prime}, n}^{z}\right) \\
& +\frac{1}{2} \sum_{r, n, n-1} J_{r, n, n-1}\left(S_{r, n} \cdot S_{r, n-1}+\sigma S_{r, n}^{z} S_{r, n-1}^{z}\right) \\
& +\frac{1}{2} \sum_{r, n, n+1} J_{r, n, n+1}\left(S_{r, n} \cdot S_{r, n+1}+\sigma S_{r, n}^{z} S_{r, n+1}^{z}\right) \\
& -g \mu_{\mathrm{B}} H \sum_{r, n} S_{r, n}^{z}+\mathcal{H}_{r, r^{\prime}, n, n-1}+\mathcal{H}_{r, r^{\prime}, n, n+1},
\end{aligned}
$$

where

$$
\begin{aligned}
& \mathcal{H}_{r, r^{\prime}, n, n-1}= \\
& \frac{1}{2} \sum_{r, r^{\prime}, n, n-1} D_{r, r^{\prime}, n, n-1}\left[S_{r, n}^{z} S_{r, n-1}^{z}\left(S_{r^{\prime}, n}^{z}\right)^{2}+S_{r, n}^{z} S_{r, n-1}^{z}\left(S_{r^{\prime}, n-1}^{z}\right)^{2}\right], \\
& \mathcal{H}_{r, r^{\prime}, n, n+1}= \\
& \frac{1}{2} \sum_{r, r^{\prime}, n, n+1} D_{r, r^{\prime}, n, n+1}\left[S_{r, n}^{z} S_{r, n-1}^{z}\left(S_{r^{\prime}, n}^{z}\right)^{2}+S_{r, n}^{z} S_{r, n+1}^{z}\left(S_{r^{\prime}, n+1}^{z}\right)^{2}\right] .
\end{aligned}
$$

Here $r$ denotes the two-dimensional position vector of spins belonging to the given layer labelled by the index $n$, where $n=1$ denotes the surface layer. The summations in (1), (2) always run over different lattice sites. The symbol $I$ denotes the ferromagnetic intralayer exchange parameter, whereas $J$ is the antiferromagnetic interlayer one. The coefficients $\sigma$ and $\sigma^{\prime}$ characterize the uniaxial two-ion anisotropy terms, and $H$ is the applied magnetic field in the $z$-direction. The terms (2) describe the three-ion anisotropic interactions between adjacent layers.

In order to perform the surface mode calculations, explicit analytical expressions for the two-dimensional Fourier-transformed temperature Green functions are found. 


\section{Numerical results}

Figure 1 presents the dispersion relations for $\mathrm{FeBr}_{2}$ in applied field $g \mu_{\mathrm{B}} H=$ $4 \mathrm{~cm}^{-1}\left(H_{\mathrm{AP}}=3.7 \mathrm{~cm}^{-1}\right)$ and for the case of the parallel wave vector along the $x$-direction. Moreover, the following values for the exchange and anisotropy parameters were taken: $I_{1}=5.07 \mathrm{~cm}^{-1}, I_{2}=-1.2 \mathrm{~cm}^{-1}, \sigma=\sigma^{\prime}=0.28$, and $D=2.45 \mathrm{~cm}^{-1}$.

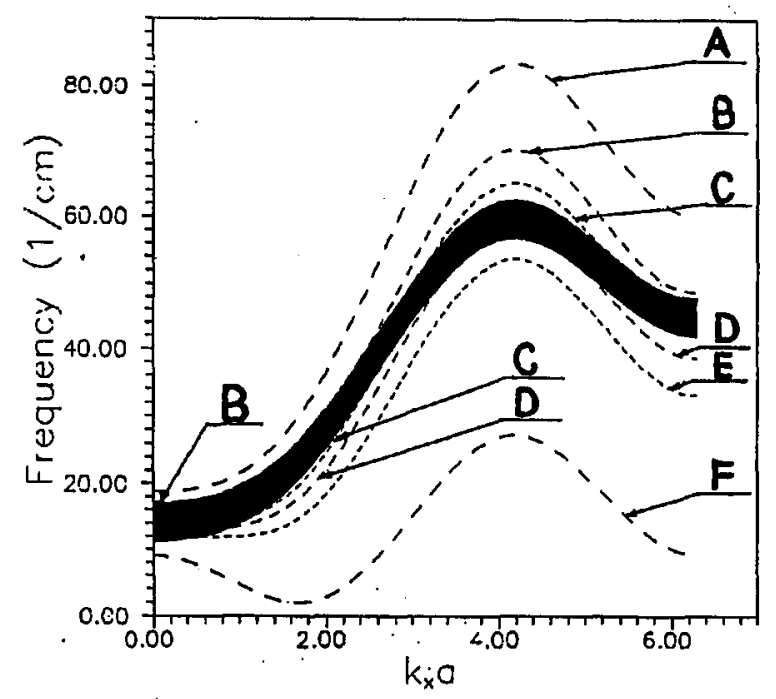

Fig. 1. Dispersion relations of bulk and surface spin waves in $\mathrm{FeBr}_{2}$ for the $\mathrm{P}$ phase, taking $g \mu_{\mathrm{B}} H=4 \mathrm{~cm}^{-1}$ for the applied field and parallel vector $k$ along the $x$-direction. The shaded area includes the bulk modes. The different surface spin-wave curves (dashed lines) correspond to A $\left(I_{1 \mathrm{~s}}=7.24 \mathrm{~cm}^{-1}\right), \mathrm{B}\left(I_{1 \mathrm{~s}}=6 \mathrm{~cm}^{-1}\right), \mathrm{C}\left(I_{1 \mathrm{~s}}=\right.$ $\left.5.5 \mathrm{~cm}^{-1}\right), \mathrm{D}\left(I_{1 \mathrm{~s}}=5.07 \mathrm{~cm}^{-1}\right), \mathrm{E}\left(I_{1 \mathrm{~s}}=4.5 \mathrm{~cm}^{-1}\right), \mathrm{F}\left(I_{1 \mathrm{~s}}=2 \mathrm{~cm}^{-1}\right)$ all with $J=$ $1.45 \mathrm{~cm}^{-1}$ and $I_{2 \mathrm{~s}}=2 \mathrm{~cm}^{-1}$.

The shaded area in Fig. 1 represents the bulk spin waves, whereas the dashed lines (A to F) represent the surface branches calculated for selected values of the $I_{1 \mathrm{~s}}$ parameter and for the case $I_{2 \mathrm{~s}}>I_{2}$ (the value of $I_{2 \mathrm{~s}}$ is the same for all $I_{1 \mathrm{~s}}$ ). Different qualitative features of SSW are as follows:

(i) the full-zone optical SSW illustrated by curve A. The acoustic full-zone SSW branches were predicted to exist for other combinations of $I_{1 \mathrm{~s}}$ and $I_{2 \mathrm{~s}}$;

(ii) the truncated SSW (curves B, E). These excitations exist only over a limited region of $k$ space.

(iii) the multitruncated SSW (curves C and D in Fig. 1). The difference between this case and the one represented by curves $B, E$ is that the surface branches intersect the bulk band more than once and they appear either in the acoustic or the optical regime depending on a competitive relation between intralayer exchange couplings;

(iv) the soft SSW (curve F). In this case the ground state of the system 
becomes unstable and the spin arrangement within the surface of our metamagnet has symmetry lower than that in the bulk of the material.

By varying both $I_{1 \mathrm{~s}}$ and $I_{2 \mathrm{~s}}$ we have constructed diagrams which show the regions in the $\left(I_{1 \mathrm{~s}}, I_{2 \mathrm{~s}}\right)$-plane where the collinear ferromagnetic arrangement of surface spins is stable, and where it is not. The results of this study are summarized

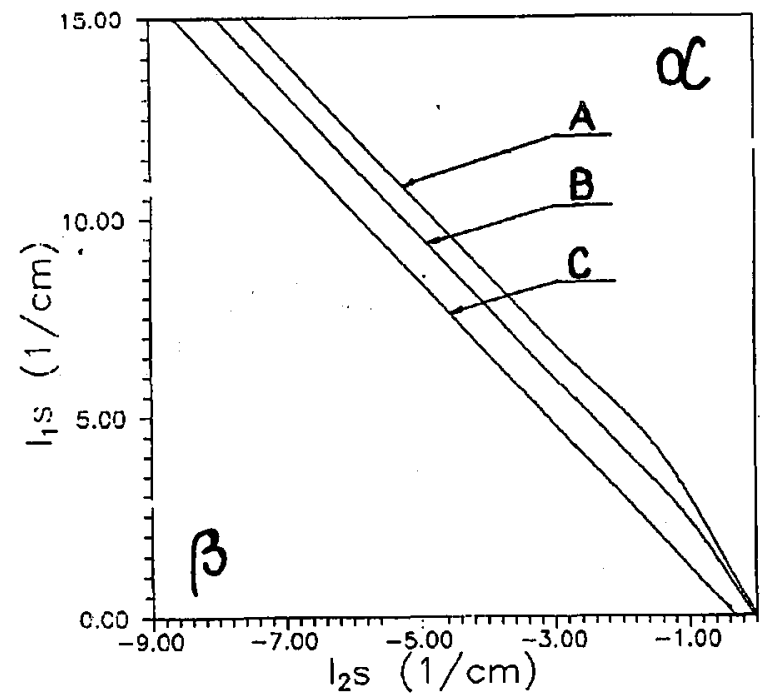

Fig. 2. Stability boundaries for the surface region, in the $\left(I_{1 s}, I_{2 s}\right)$-plane. The surface spin stability area and the surface instability area is denoted by $\alpha$ and $\beta$, respectively. The different lines correspond to A $\left(J=0.5 \mathrm{~cm}^{-1}\right), \mathrm{B}\left(J=1.45 \mathrm{~cm}^{-1}\right)$ and $\mathrm{C}(J=$ $3 \mathrm{~cm}^{-1}$ ).

in Fig. 2; the critical boundaries dividing the ( $\left.I_{1 \mathrm{~s}}, I_{2 \mathrm{~s}}\right)$-plane into the surface spin stability and the surface spin instability area are plotted for different values of the $J$ parameter. Thus, we have found that for $J$ values small enough, the ground state of the system is determined by the surface frequencies touching both the $\omega_{\mathrm{s}}(k=0)$ (the full-zone acoustic SSW) and $\omega_{\mathrm{b}}[k=(0,0, \pi)]$ (bottom of the bulk band) values. This case is illustrated in lines A and B in Fig. 2. With increasing $J$ the system becomes, as expected, more stable and simultaneously in lines from Fig. 2, the number of critical points corresponding to the ground state energy being equal to $\omega_{\mathrm{b}}[k=(0,0, \pi)]$ increases. The stability line $\mathrm{C}$ in Fig. 2 presents the case when the ground state is determined only by the bottom of the bulk band.

\section{Final remarks}

The results obtained in this paper as well as the results of our earlier studies $[2,3]$ provide rich material for experimental verification, e.g. by light-scattering techniques. The critical lines in Fig. 2 together with different types of dispersion relations indicate the area where one should expect surface spin stability when 
searching for SSW lying in the acoustic or optical regime. Moreover, these results permit establishing conditions for a sample to provide the splittings of SSW above or below the bulk modes sufficiently large for experimental detection.

On the theoretical side, such problems as the quantitative description of the reconstruction and calculating light-scattering intensities are still open to discuss.

\section{References}

[1] J.H. Baskey, M.G. Cottam, Phys. Rev. B 42, 4304 (1990).

[2] W. Rudziński, W. Maciejewski, Phys. Status Solidi B 174, 547 (1992).

[3] W. Rudziński, W. Maciejewski, Phys. Status Solidi B 175, 247 (1993).

[4] Z. Onyszkiewicz, A. Wierzbicki, Physica B+C 151, 475 (1988). 